to place it under a microscope. Any ideas welcomed. Roy Beavers rbeavers@mail.smu.edu Thu Jun 26

I've found tiny pieces can jump feet away from microscopes. Some I have yet to find! How far something goes depends on the pressure on the forceps and the angle of the hand holding them. I doubt that a small tray would catch most fragments. I would think a curtain that surrounds the sides and back of the scope would stop the object from going further then add a large ridged or slightly sticky rubber mat on the counter to catch the piece before it rolled further. That should catch about $75 \%$. How about prevention? Could you use a single chambered slide to capture the piece before putting it on the scope stage? That way it would not be lost if the slide jumped a bit as it was put on the scope stage. Borrow an inverted microscope from the Life Sciences Department if the magnification requirement is equal. Working from the top is much easier to do. Another thought...a small piece of double sticky tape on the slide with the object pushed up against the side of the tape. Not good if using high power since the objective would also stick when it got close to the piece of interest. Just about anything that avoids using the forceps at such an angle as is necessary to get something under an objective would be helpful. All sorts of things are going through my brain like a thin coating of honey on the slide so the tooth could be put on easily, stick during observation, easily washed clean with water... "Small tray with side walls" - a square Petri dish? What size are you thinking about? Patricia Stranen Connelly connellyps@ nhlbi.nih.gov Thu Jun 26

Why not go to your local photography dealer and get an appropriate size of photo paper processing tray. These have many non-photo uses in our lab at Kodak. The largest ones (or 'liberated' cafeteria trays ) are great to hold rotary vacuum pumps that always choose the most inopportune moment to dump oil all over the lab. J.R. Minter jrminter@rochester.rr.com Thu Jun 26

Another alternative might be to get a small pump, which not only blows but sucks. You can get a variety of attachments to hold tiny things, or make them yourself. We use ours mainly as an air jet for cleaning things, but we can attach the hose to the other end and get suction. Rosemary White rosemary.white@csiro.au Thu Jun 26

Handling TEM specimens with tweezers is not advisable. I also have had similar bad experiences in the past. The best way is to handle them vacuum pipette. You can buy 18 " needles from SPI as

\section{The Ghost of the IEL: A Halloween Photoshop Exercise}

\section{Emily Bradford ${ }^{\star}$ Gary Shull, ${ }^{\star}$ and MarianMiller ${ }^{\star \star}$ \\ ${ }^{*}$ Dept. of Molecular Genetics, ${ }^{*}$ Dept. of Environmental Health, Univ. of Cincinnati,Cincinnati $\mathrm{OH}$.}

\section{millermn@email.uc.edu}

Image of an intraepithelial lymphocyte (IEL) from a CLIC5 mutant mouse small intestine. The CLIC (Chloride Intracellular Channel) family of proteins is expressed in a wide variety of cell types, and several isoforms are known to cycle between soluble and membranebound forms (Cromer et al. 2002). As well as being widely expressed, the CLICs are involved in diverse functions, including tubulogenesis (Berry et al.2003), immune cell activation, apoptosis (Suh et al. 2005) and calcium handling (Board et al. 2004). CLIC5 has been shown to

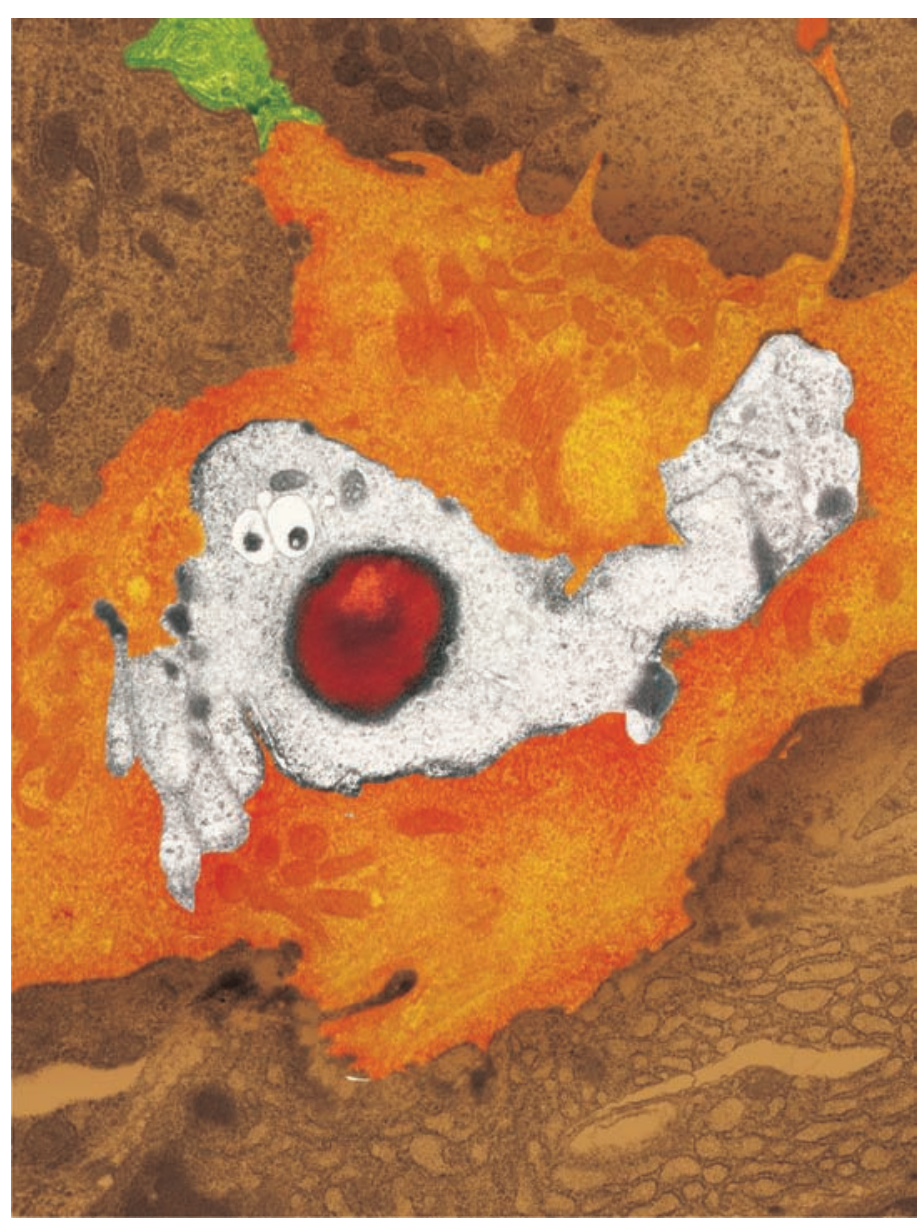

associate with cytoskeletal proteins in placental microvilli and inner ear cells, and is required for proper maintenence of hair cell steriocilia (Berryman et al. 2000; Gagnon et al. 2006). It has also been localized to the cytosol of human intestinal epithelial cells, though its function there remains unclear. The study in which this particular "IEL" was found, involved a search to see what function CLIC5 played in the modulation of tubulovesicles and microvillar apical membranes in the process of acid secretion. In particular, the relative amounts and structural characteristics of these two membrane types was quantified in parietal cells.

The pseudo-colored image was created as follows: The original electron micrograph was converted to RGB. The image of "Casper, the friendly ghost" was prepared in Photoshop. Separate layers of electron micrograph were cut with the lasso tool, to include the ghost, the pumpkin the stem and the background. For each layer, a foreground color was chosen, then the layer was selected (AltS $>A$ ), and a gradient map was applied (Image $>$ Ajustments $>$ Gradient map. Double clicking on the gradient map image allowed for selecting additional colors and editing the gradient. The bandaid tool was used to remove dust and scratches, the burn and dodge tools were used to create the drop shadow effect and give a 3-D effect to the objects. The photoshop image was imported into Corel Draw $\mathrm{x} 3$, and power clipped into the appropriate sized rectangle and exported as a tiff (CMYK at $300 \mathrm{dpi}$ ).

Berry et al. Science. 2003 Dec 19;302(5653):2077-8.

Berryman et al. Mol Biol Cell. 2000 May;11(5):1509-21.

Board et al. Int J Biochem Cell Biol. 2004 Aug;36(8):1599-612.

Cromer et al. Eur Biophys J. 2002 Sep;31(5):356-64

Gagnon et al. J Neurosci. 2006 Oct 4;26(40):10188-98.

Suh et al. J Investig Dermatol Symp Proc. 2005 Nov;10(2):105-9. 